\title{
IDENTIFICATION OF RADIOACTIVE ISOTOPES USING CHOLESKY-DECOMPOSITION OF MATRICES
}

\author{
László HANKA \\ Óbuda University, Budapest, Hungary \\ hanka.laszlo@bgk.uni-obuda.hu
}

\begin{abstract}
The process of identification of radioactive isotopes using gamma ray spectrum produced by scintillation detectors is a fundamental problem in physics. Military applications also require fast and efficient methods, especially in field conditions, for identifying unknown isotopes. The fundamental problem is the relationship between the observed gamma ray spectrum given by the detector and the real spectrum. This problem can be treated as a mathematical problem. The relationship between the real and the observed spectrum can be described by a linear algebraic equation system. In this article an efficient mathematical procedure is proposed to solve this linear system efficiently.
\end{abstract}

KEYWORDS: gamma ray spectrum, deconvolution, Matlab Cholesky-factorization, regularization

\section{Introduction}

The structure of gamma ray spectrum, produced by a scintillation detector can be quite complicated (Bouchet, 1995; Jandel et al., 2004; Meng \& Ramsden, 2000). For example an isotope, that emits only one gamma line, like Caesium-137 $\left({ }^{137} \mathrm{Cs}\right)$, that emits a single gamma line of $662 \mathrm{keV}$, the observed gamma ray spectrum, produced by a scintillation detector with $\mathrm{CsI}(\mathrm{Tl})$ (Thallium activated Cesium Iodide) scintillation crystal, can be seen on Figure no. 1.

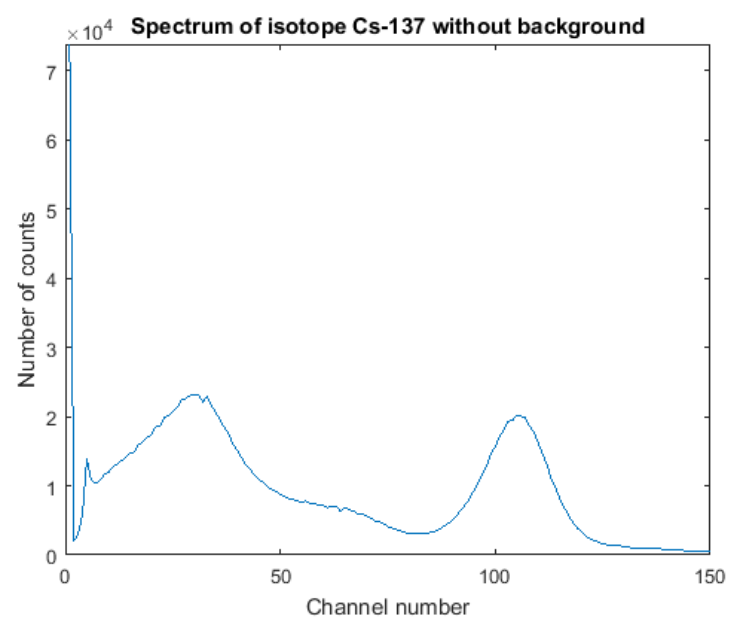

Figure no. 1: The observed gamma spectrum of ${ }^{137} \mathrm{Cs}$ 
The observed spectrum is a continuous spectrum, with three characteristic peaks, thanks to physical processes in the scintillator (Gopinath \& Gopala, 1999; Morhac, 2006). The peak on the right end, the full energy peak, is the photo peak that directly corresponds to the single gamma line. The low energy peak is the X-ray emission of internal conversion. Between these peaks there is the Compton region, due to Compton scattering it is continuous, and at the low energy end of the Compton distribution the backscatter peak can be seen.

This spectrum has been registered by a detector, in which the number of channels of the multichannel analyzer is 512. Since between channels 150 and 512 the number of counts practically was zero, only the important part of the spectrum is illustrated on Figure no. 1.

In real measurements the background radiation must be taken into account. Obviously the spectrum of the background was registered separately, without any studied radioactive source, and Figure no. 1 illustrates only the spectrum of the studied isotope, the spectrum of the background was subtracted.

In the following example, the isotope Cobalt-60 $\left({ }^{60} \mathrm{Co}\right)$ is studied, that emits two gamma lines of $1,17 \mathrm{MeV}$ and $1,33 \mathrm{MeV}$ respectively. On Figure no. 2 the observed spectrum can be seen, produced by the same detector. Only the important part of the spectrum is illustrated again without background.

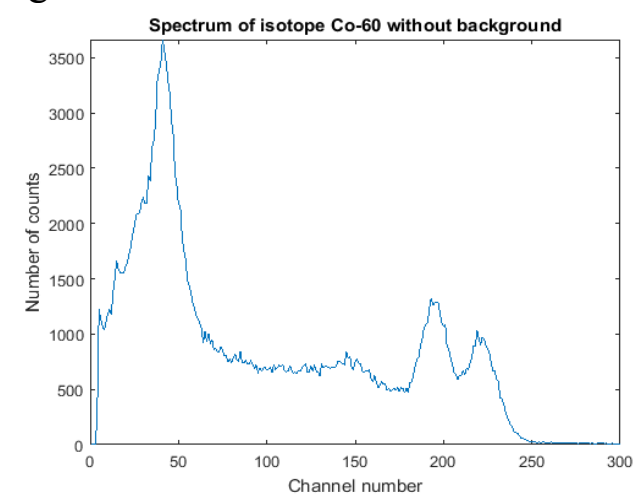

Figure no. 2: The observed gamma spectrum of ${ }^{60} \mathrm{Co}$
The separated photo peaks can be seen at the high energy end of the spectrum. These peaks correspond to the gamma lines respectively. At the low energy end the backscatter peak can be seen.

As it can be seen on previous figures, the observed spectrum can be rather complex, however the corresponding real spectrum is pretty simple, contains only one or two gamma lines.

If a radioactive source contains several different nuclides, with lots of gamma lines, and different intensities, anyone can imagine, how complicated the registered spectrum will be. A basic problem in radiology, using the gamma spectrum, produced by a detector, giving the real spectrum, the list of real gamma lines. If energy of real gamma lines is known, using a handbook, radionuclides can be identified. Mathematics can help to solve this problem.

\section{Deconvolution}

The problem described in the introduction can be considered as a mathematical problem (Gerasimov, 1995; Meng \& Ramsden, 2000; Los Arcos, 1996). The first step is giving the real spectrum as a vector, denoted by $\mathbf{x}$. The number of coordinates of this vector equals to the number of channels of the analyzer, which is 512 in this study. There is a correspondence between cannel number $c$ and the energy of the gamma ray $E$. The most simple assumption is that the energy is proportional to the number of channels. For the sake of better accuracy in this study a quadratic polynomial was fitted for describing this correspondence. The form of the polynomial is:

$$
E=a_{1}+a_{2} \times c+a_{3} \times c^{2}
$$

Using observed spectrum of some radioactive sources $\left({ }^{137} \mathrm{Cs},{ }^{60} \mathrm{Co},{ }^{22} \mathrm{Na}\right.$, ${ }^{133} \mathrm{Ba},{ }^{241} \mathrm{Am},{ }^{85} \mathrm{Sr}$, etc.) and using least squares method for finding the best fit parabola, the unknown coefficients can be 
determined. In this study these constants are the following:

$$
\begin{aligned}
& a_{1}=-9,047 \times 10^{-3} \\
& a_{2}=6,0110 \times 10^{-3} \\
& a_{3}=2,5215 \times 10^{-7}
\end{aligned}
$$

This procedure is called energy calibration. So, thanks to this function, for every cannel the corresponding gamma energy can be determined, and since that over the studied energy region this parabola is invertible, for every specific energy the corresponding channel number also can be given. So the real spectrum can be modeled by the above defined $\mathbf{x}$ vector. A coordinate of this vector, if its channel number corresponds to the specific gamma energy according to energy calibration, is proportional to the intensity, for example equals to the number of counts, and any other coordinate is zero.

The observed spectrum is given by another vector, denoted by $\mathbf{y}$, which coordinates are number of counts in every specific channel between 1 and 512 .

So $\mathbf{x}$, the real spectrum is the input, and $\mathbf{y}$, the observed spectrum is the output. This correspondence mathematically can be described by a linear map, more precisely by a matrix, which is denoted by $\mathbf{R}$. This is the response matrix of the detector (Berger \& Seltzer, 1972; Shi, Chen, Li \& Yun, 2002; Pokoradi, 2016). The response matrix is quadratic, real, and its size is $512 \times 512$ in this study.

The linear transform is given by the linear equation system:

$$
\mathbf{R x}=\mathbf{y}
$$

Assuming that the response matrix $\mathbf{R}$ of the detector is known, and that the observed spectrum $\mathbf{y}$ is also known, the fundamental mathematical problem is solving this linear system. But this is absolutely not trivial problem. The first idea could be taking the inverse of $\mathbf{R}$, and multiplying vector $\mathbf{y}$ by the inverse:

$$
\mathbf{x}=\mathbf{R}^{-1} \mathbf{y}
$$

Unfortunately this operation doesn't work, that is illustrated below. For calculations Matlab software has been used, which is a very efficient tool for solving such problems. Applying this operation for the ${ }^{60} \mathrm{Co}$ spectrum, illustrated on Figure no. 1, the Matlab answer is the following:

"Matrix is close to singular or badly scaled.

Results may be inaccurate.

RCOND $=3.469278 \mathrm{e}-44 . "$

In this warning RCOND is the reciprocal of the condition number of matrix R. This condition number is extremely big now, and due to this fact the solution is unreliable (Stoer \& Burlish, 1991). Because by definition the condition number is the ratio of the greatest and the smallest eigenvalue:

$$
\kappa=\operatorname{cond}(\mathrm{R})=\lambda_{\max } / \lambda_{\min }
$$

In numerical mathematics it is proved, that the condition number affects the sensitivity of the system to measurement error, according to the following formula:

$$
\frac{\|\delta \mathrm{x}\|}{\|\mathrm{x}\|} \leq \kappa \frac{\|\delta \mathrm{y}\|}{\|\mathrm{y}\|}
$$

This formula means that if $\delta \mathbf{y}$ is the absolute error of measurement, than the proportional error of the solution, of the real spectrum in this case can be $\kappa$-times more than the proportional error of the measurement. In this case, the condition number of the response matrix is approximately $\sim 10^{40}$, therefore it is clear, that the solution can't be accurate. The solution is extremely sensitive to measurement error. On Figure no. 3 the extremely oscillating "deconvolved spectrum" can be seen when the formula $\mathbf{x}=\mathbf{R}^{-1} \mathbf{y}$ is used. It is clear, that this solution makes no sense at all. 


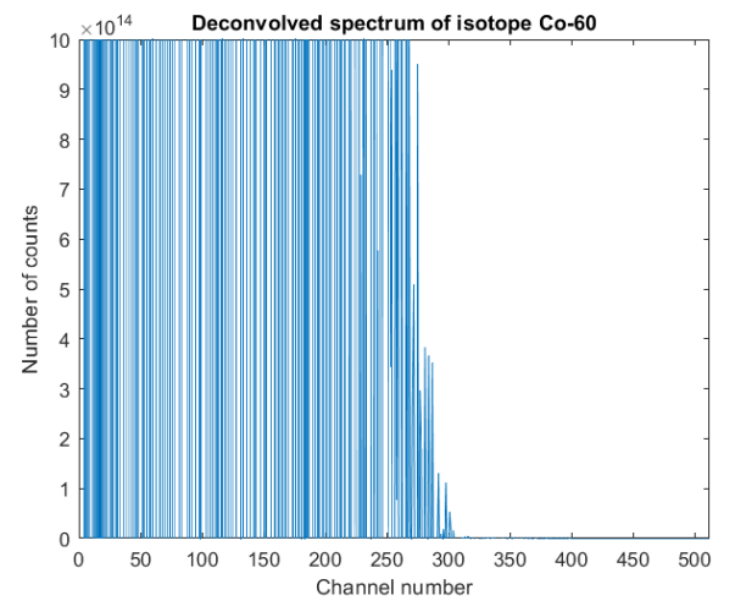

Figure no. 3: The effect of extreme big condition number

Consequently a completely different procedure must be applied for giving the real spectrum.

\section{The Gaussian Equation}

Instead of solving the system, and giving the accurate solution, which might not exist, one possible procedure can be giving the best approximate solution. In this case this problem is considered as a optimization problem (Bouchet, 1995; Kiss, Pintér \& Szakács, 2017; Morhac, 2006). In general if the system

$$
\mathbf{A x}=\mathbf{y}
$$

has no solution, the best approximate solution of this unsolvable system can be given by the solution of the system

$$
\mathbf{A}^{\mathrm{T}} \mathbf{A} \mathbf{x}=\mathbf{A}^{\mathrm{T}} \mathbf{y}
$$

which is the so-called Gaussian equation (Halmos, 1958; Strang, 2003). Superscript " $T$ " refers to the transpose of the matrix. The advantage of this system is, that the coefficient matrix, $\mathbf{A}^{\mathrm{T}} \mathbf{A}$ is quadratic, symmetric and positive semi definite, which means that every eigenvalue is non negative. In linear algebra it turns out, that symmetric matrices are the "best" matrices, they have lots of good properties, so there is a hope to solve the original problem by this transformation.
Applying this theory to the studied problem, a possible algorithm will be proposed to solve the Gaussian system

$$
\mathbf{R}^{\mathrm{T}} \mathbf{R} \mathbf{x}=\mathbf{R}^{\mathrm{T}} \mathbf{y}
$$

for finding the real spectrum $\mathbf{x}$. In the following section a specific tool, a factorization procedure will be applied for the symmetric coefficient matrix.

\section{Cholesky Decomposition}

The Cholesky-decomposition or Cholesky-factorization is a very efficient tool in linear algebra (Strang, 2003). This decomposition is mainly used for solving linear systems of the form

$$
\mathbf{A x}=\mathbf{y}
$$

where $\mathbf{A}$, the coefficient matrix is symmetric.

The Cholesky-factorization is a specific case of the most general LU decomposition of matrices. LU decomposition is the matrix-multiplication form of Gaussian elimination procedure, and can be applied for any matrix. Any matrix $\mathbf{A}$ can be given in the product form

$$
\mathbf{A}=\mathbf{L} \mathbf{U}
$$

where $\mathbf{L}$ is a lower triangular quadratic matrix, and $\mathbf{U}$ is a general upper triangular matrix. In matrix $\mathbf{L}$ every entry in the main diagonal is one, and above the main diagonal every entry is zero. This factorization is used in numerical mathematics to solve systems.

But if the coefficient matrix is symmetric, the factorization is specific. Assume, that the coefficient matrix is symmetric, which means that $\mathbf{A}=\mathbf{A}^{\mathrm{T}}$. In this case, the factorization of the coefficient matrix can be given in the form

$$
\mathbf{A}=\mathbf{L L}^{\mathrm{T}}
$$

This factorization is called Cholesky factorization (Strang, 2003). In this product the second factor is the transpose of the first factor. Comparing Cholesky-decomposition and the LU factorization, this is the 
fundamental difference. If the matrix $\mathbf{A}$ is positive definite, this factorization is unique, and every entry in the main diagonal of $\mathbf{L}$ is strictly positive. If matrix A is "only" positive semidefinite, the matrix L needn't be unique, and some entries in the main diagonal can be zero.

The application of the Choleskydecomposition for linear system is the following. In the system $\mathbf{A x}=\mathbf{y}$, assuming that the coefficient matrix $\mathbf{A}$ is symmetric, A is replaced by the decomposed form

$$
\mathbf{L L}^{\mathrm{T}} \mathbf{x}=\mathbf{y}
$$

and the system can be solved in two steps. On the one hand, using $\mathbf{L}$ and $\mathbf{y}$ the system

$$
\mathbf{L z}=\mathbf{y}
$$

is solved for $\mathbf{z}$, and on the other hand, using $\mathbf{L}^{\mathrm{T}}$ and $\mathbf{z}$ the system

$$
\mathbf{L}^{\mathrm{T}} \mathbf{x}=\mathbf{z}
$$

is solved for $\mathbf{x}$. The structure of these systems is very simple, since coefficient matrices are triangular in both cases, which means that only the back substitution procedure must be applied for solution, in other words the procedure is pretty simple. According to experiences in numerical mathematics, the Cholesky - decomposition is more efficient than the simple LU decomposition. Therefore an advantageous situation, if the coefficient matrix is symmetric. There is one more advantage of this factorization, namely the inverse of the matrix needn't be determined.

In this study, the Cholesky factorization is applied, under appropriate conditions, for giving the real spectrum. For calculations Matlab software was used, because in Matlab the Cholesky decomposition can be produced by one simple command.

\section{Regularization}

In the Gaussian system

$$
\mathbf{R}^{\mathrm{T}} \mathbf{R} \mathbf{x}=\mathbf{R}^{\mathrm{T}} \mathbf{y}
$$

the coefficient matrix $\mathbf{R}^{\mathrm{T}} \mathbf{R}$ "only" positive semi definite in general, which means, that some of the eigenvalues are zero. If a matrix ha zero eigenvalue, it means that the matrix is singular, in other words, not invertible (Strang, 2003). If the coefficient matrix is singular, the condition number is extremely big, consequently the system can't be solved accurately.

Regularization procedure transforms the positive semi definite matrix into positive definite matrix (Bouchet, 1995; Morhac, 2006). The operation is the following in general: in the system the coefficient matrix $\mathbf{A}$ is replaced by matrix

$$
\mathbf{A}+\alpha \mathbf{I}
$$

where $\mathbf{I}$ is the identity matrix, $\alpha$ is the regularization parameter, which is a small positive real number. If $\alpha$ is small enough, approximately between $10^{-7}$ and $10^{-9}$ in this study, the difference between $\mathbf{A}$ and $\mathbf{A}+\alpha \mathbf{I}$ is negligible, therefore this transformation doesn't affect the solution essentially. But the direct consequence of this modification, that if $\mathbf{A}$ is positive semidefinite, $\mathbf{A}+\alpha \mathbf{I}$ is positive definite, consequently nonsingular. Namely, assuming that $\lambda$ is an eigenvalue of $\mathbf{A}$ and $\mathbf{v}$ is the corresponding eigenvector, the following holds:

$(\mathbf{A}+\alpha \mathbf{I}) \mathbf{v}=\mathbf{A v}+\alpha \mathbf{I} \mathbf{v}=\lambda \mathbf{v}+\alpha \mathbf{v}=(\lambda+\alpha) \mathbf{v}$

The meaning of this equation, that the new eigenvalue is strictly positive: $\lambda+\alpha>0$. So the matrix, obtained by the regularization procedure is indeed positive definite. Applying this observation to the studied system, instead of the original Gaussian system, the following modified Gaussian system must be solved:

$$
\left(\mathbf{R}^{\mathrm{T}} \mathbf{R}+\alpha \mathbf{I}\right) \mathbf{x}=\mathbf{R}^{\mathrm{T}} \mathbf{y}
$$

Since $\mathbf{R}^{\mathrm{T}} \mathbf{R}$ is symmetric, and the identity matrix is also symmetric, the coefficient matrix $\mathbf{R}^{\mathrm{T}} \mathbf{R}+\alpha \mathbf{I}$ is a symmetric, positive definite coefficient matrix. For this matrix, for this regularized system the Cholesky-factorization will be applied in order to find an accurate solution. 
Introducing a new notation for the sake of simplicity, the coefficient matrix will be denoted simply by $\mathbf{A}$, so

$$
\mathbf{A}=\mathbf{R}^{\mathrm{T}} \mathbf{R}+\alpha \mathbf{I}
$$

therefore the system to be solved is the following:

$$
\mathbf{A x}=\mathbf{R}^{\mathrm{T}} \mathbf{y}
$$

There is one more requirement that must be taken into account. Negative solutions can't be accepted, since the meaning of one coordinate of the solution vector $\mathbf{x}$ is the number of counts in one specific channel. This is the reason why the previously described regularization procedure must be modified again.

\section{Wighted Regularization}

The acceptable solution will be produced by an iteration procedure. In the zeros step the system

$$
\mathbf{A x}=\mathbf{R}^{\mathrm{T}} \mathbf{y} ; \quad \mathbf{A}=\mathbf{R}^{\mathrm{T}} \mathbf{R}+\alpha \mathbf{I}
$$

is solved using $\mathbf{y}^{(0)}=\mathbf{y}$ as an initial value of the iteration, and then in general in the $k$ th step a modified system

$$
\begin{gathered}
\mathbf{A} \mathbf{x}^{(\mathrm{k})}=\mathbf{R}^{\mathrm{T}} \mathbf{y}^{(\mathrm{k})} ; \mathbf{y}^{(\mathrm{k}+1)}=\mathbf{R} \mathbf{x}^{(\mathrm{k})} ; \\
\mathbf{A}=\mathbf{R}^{\mathrm{T}} \mathbf{R}+\alpha \mathbf{Q}
\end{gathered}
$$

is solved, where matrix $\mathbf{Q}$ is a modified identity matrix. The definition is the following: The $q_{i i}$ entry in the main diagonal is one, if $x_{i}$ is non negative in the $k$ th step, and equals to some $\beta$ "weight parameter" if $x_{i}$ coordinate is negative. Every other entry of $\mathbf{Q}$ is still zero. According to our experiences, this weighted regularization works efficiently, if the value of $\beta$ is between 100 and 10000 . Using this method the number of negative coordinates can be reduced in every iteration step.

In every step of the described iteration the Cholesky-factorization is applied in two steps, as it was mentioned in section 4. The only disadvantage of this procedure, that the coefficient matrix

$$
\mathbf{A}^{(\mathrm{k})}=\mathbf{R}^{\mathrm{T}} \mathbf{R}+\alpha \mathbf{Q}^{(\mathrm{k})}
$$

changes in every iteration step, due to negative coordinates of $\mathbf{x}$, therefore the calculation of Cholesky-decomposition, the procedure requires lots of computations in every step. But, as it will be illustrated in the following sections, the procedure is efficient.

\section{Spectrum \\ 7. Application for an Artificial}

Testing the efficiency of the proposed algorithm an ideal gamma spectrum was constructed using only pure Gaussian curves, at channel positions 90, 140, 200, 250, 300, 400. One Gussian curve can be an ideal response for a single gamma line.

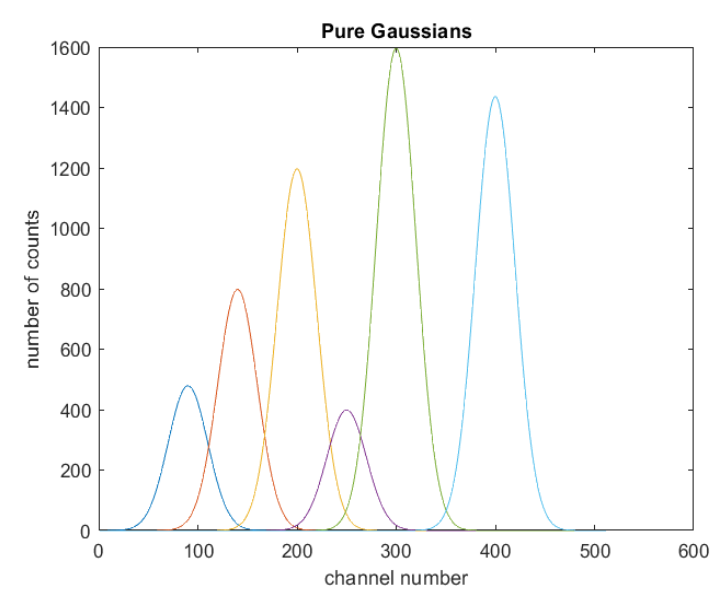

Figure no. 4: Pure Gaussian curves, as photo peaks

These pure Gaussians can be seen on Figure no. 4 separately. These Gaussian curves can be considered as spectrum of pure photo peaks. The superposition of these curves, the artificial spectrum is illustrated on Figure no. 5. This curve is a simple model of a complex gamma spectrum. 


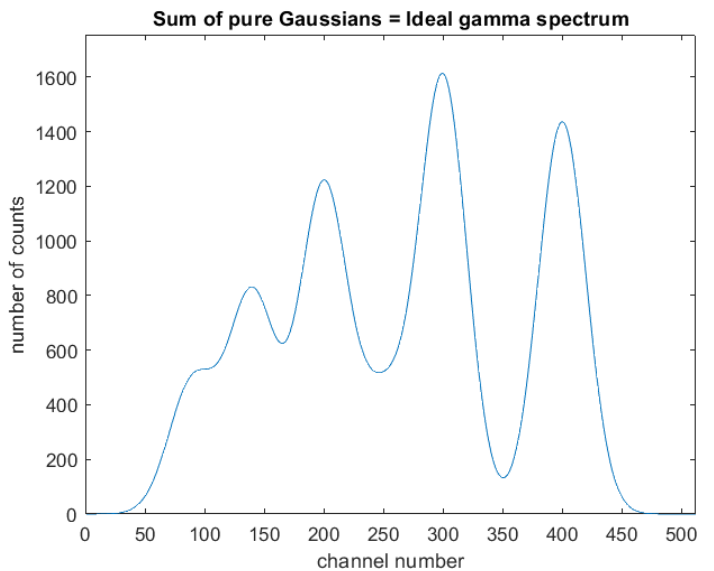

Figure no. 5: Sum of Pure Gaussians, an ideal spectrum

The procedure can be tested using this ideal spectrum. The basic question: does this algorithm provide every single gamma line, including the fourth one at $c=250$, which is absolutely suppressed by the other five gamma lines, so is absolutely not detectable on Figure no. 5. And the second and as important question as the previous: does this algorithm provide the exact positions of gamma lines, or not.

The proposed algorithm was applied to the complex artificial spectrum.

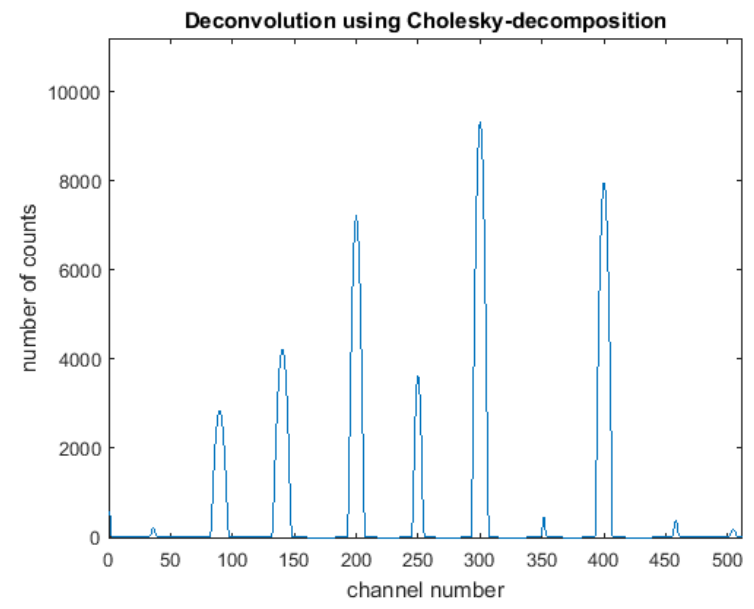

Figure no. 6: Application of the algorithm for the artificial spectrum

The regularization and weight parameters has been chosen as $\alpha=10^{-9}$, $\beta=1000$ respectively. The number of iteration steps was 1000 , and the total procedure took only less than 10 seconds.
The result of the deconvolution is illustrated on Figure no. 6. Taking a look at Figure no. 6, it is clear, that every single gamma line, even the fourth one in the background, can be identified, and also positions of gamma lines are accurate. In practice, obviously, a more sophisticated method is chosen for identifying the position of peaks: a Gaussian curve must be fitted for every peak, and the fitted mean value will be the position of the peak. This more accurate method is applied in the following section.

\section{Application for Real Spectrum}

Testing the proposed algorithm for real spectrum, the spectrum of mixed nuclides, Co-60 and Cs-137 was chosen. The spectrum of this mixture can be seen on Figure no. 7.

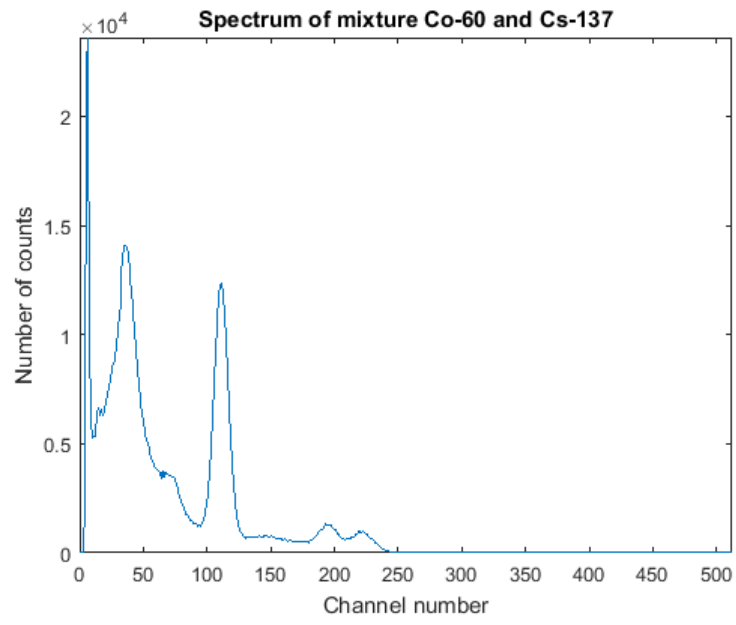

Figure no. 7: Spectrum of the mixture

For this spectrum the procedure has been applied. The regularization and weight parameters has been chosen similarly, like in the previous artificial example, $\alpha=10^{-9}$, $\beta=1000$ respectively. The number of iteration steps was again 1000, and the total procedure took only about 8 seconds. The result of the deconvolution procedure can be seen on Figure no. 8 . 


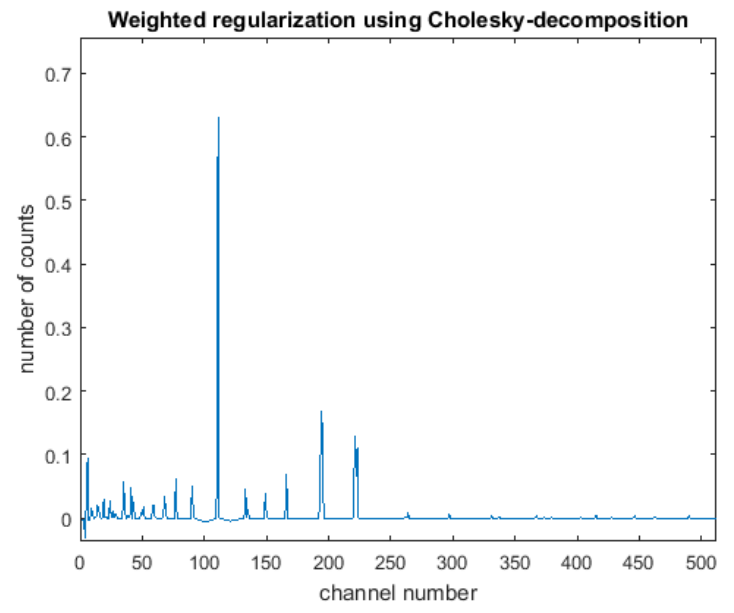

Figure no. 8: Result of the deconvolution of the Spectrum of the mixture on Figure no. 7

Three characteristic gamma lines can be seen immediately on the picture. Fitting Gaussian distributions for these three sharp peaks, identifying the channel as the mean of the distribution, and finally using energy calibration polynomial, for energy of the most characteristic peaks the following is obtained:

\begin{tabular}{|l|l|l|l|}
\hline Isotope & \multicolumn{1}{|l|}{ Co-60 } & Cs-137 \\
\hline exact & $1,1732 \mathrm{MeV}$ & $1,3325 \mathrm{MeV}$ & $0,6616 \mathrm{MeV}$ \\
\hline algorithm & $1,1727 \mathrm{MeV}$ & $1,3439 \mathrm{MeV}$ & $0,6612 \mathrm{MeV}$ \\
\hline
\end{tabular}

Comparing the exact energies of gamma lines and the energies obtained by the decomposition procedure, it is clear that these isotopes can be identified easily. The procedure works.

\section{Conclusions}

In this study an efficient and rather simple algorithm is proposed for identifying gamma sources. As it was proved in section 8 and 9, the Cholesky-factorization of matrices, extended by weighted regularization proved to be efficient tool for solving the ill-posed linear system, which is fundamental, is gamma spectroscopy. For these calculations Matlab software is recommended due to built in functions. In this study every calculation and every Figure has been made using Matlab.

\section{REFERENCES}

Berger, M. J., \& Seltzer, S. M. (1972). Response function for sodium iodine scintillation detectors. Nuclear Instrumens and Methods, Vol.104, Issue 2, 317-332.

Bouchet, L. (1995). A comparative study of deconvolution methods of gamma-ray spectra. Astronomy and Astrophysics Supplement Series, Series 113, 167-183.

Gerasimov, S. A. (1995). A direct method for separating peak multiplet in X-ray and gamma-ray spectra. Applied radiation and isotopes, Vol. 46, Issue 3, 181-184.

Gopinath, D. V., \& Gopala, K. (1999). Analitical comutation of the Compton continuum in gamma-ray spectrometry. Radiation Physics and Chemistry, Vol. 56, Issue 5-6, 525-534.

Halmos, P. R. (1958). Finite-dimensional vector spaces. Princeton, NJ: Van Nostrand.

Jandel, M., Morhac, M., Kliman, J., Krupa, L., Matousek, V., Hamilton, J. H., \& Ramaya, A. V. (2004). Decomposition of continuum $\gamma$-ray spectra using syhthesized response matrix. Nuclear Instruments \& Methods in Phisics Research, Vol. 516, Issue 1, 172-183.

Kiss, Gy., Pintér, P., \& Szakács, T. (2017). Vehicle Frame Optimization using Finite Element Method. Proceedings of the $1^{\text {st }}$ Agria Conference on Innovative Pneumatic Vehicles - ACIPV 2017. Budapest, Eger: Magyarország: Óbuda University, 66-70. 
Los Arcos, J. M. (1996). Gamma-ray spectra deconvolution by maximum-entropy methods. Nuclear Instruments and methods in Physics Research, Vol. 27, Issue 10, 634-636.

Meng, L. J., \& Ramsden, D. (2000). An Inter-comparison of Three SpectralDeconvolution Algorithms for Gamma-ray Spectroscopy. IEEE Transactions on Nuclear Science, Vol. 47, Issue 4.

Morhac, M. (2006). Deconvolution methods and their applications in the analysis of gamma-ray spectra. Nuclear Instruments and Methods in Physics Research, Vol. 559, 119-123.

Pokorádi, L. (2016). Availability assessment with Monte-Carlo simulation of maintenance process model. Politechnical University of Bucharest. Scientific Bulletin. Series D. Mechanical Engineering, Vol. 78, Issue 3, 43-54.

Shi, H., Chen, B., Li, T., \& Yun, D. (2002). Precise Monte Carlo simulation of gamma ray response function for an $\mathrm{NaI}(\mathrm{Tl})$ detector. Applied radiation and isotopes, Vol. 57, Issue 4, 517-524.

Stoer, J., \& Burlish, R. (1991). Introduction to Numerical Analysis. Berlin Heidelberg: Spinger-Verlag.

Strang, G. (2003). Introduction to Linear Algebra. MIT: Wellesley-Cambridge Press. 